\title{
Effects of SUSY, Little Higgs, etc on B decay
}

\section{Benjamín Grinstein ${ }^{* \dagger}$}

UCSD - Department of Physics, La Jolla, CA 92093-0319, USA

E-mail: bgrinstein@ucsd.edu

Flavor Changing Neutral Currents severely constrain extensions of the standard model. We review the Minimal Flavor Violation hypothesis and how, when incorporated in a low energy effective field theory description of new physics, it successfully addresses the large neutral currents "flavor problem." We then argue that, but for a few notable exceptions, models of new physics that successfully address this flavor problem lead to a low energy description that incorporates the Minimal Flavor Violation hypothesis.

Flavor Physics and CP Violation 2009,

May 27 - June 1, 2009,

Lake Placid, NY, USA

\footnotetext{
* Speaker.

${ }^{\dagger}$ Work supported in part by DOE under contract DE-FG03-97ER40546.
} 


\section{Introduction}

Don't blame me: The title of this talk was assigned to me. Also, I was instructed "etc" does not include 5-dimensional models. ${ }^{1}$ In thinking about the topic it became apparent that I could not comprehensively review what the minimal supersymmetric extension of the standard model (MSSM) and its variants have to say about $B$-decays, let alone other 4-dimensional extensions of the Standard Model (SM). The subject is too vast. Worse, peculiarities of models are often ad hoc and it is these details that make the subject so vast. Instead I will try to find some common threads and see if we can make some generic claims of what classes of models imply for $B$-decays.

To this end let us contrast the "bottom-up" to the "top-down" approaches to New Physics (NP, also BSM). Since the NP is not yet directly accessible to experiment its effects appear indirectly as modifications to SM interactions among SM particles. For the bottom-up approach we assume these interactions can be described by supplementing the SM Lagrangian with terms of dimension higher than four ("higher dimension operators") as allowed by Lorentz Invariance and gauge symmetries. A term of dimension $n>4$ appears in the Lagrangian with coefficient $c / \Lambda_{\mathrm{NP}}^{n-4}$. The parameter $\Lambda_{\mathrm{NP}}$ has unit mass dimensions and characterizes the energy scale of the NP. Hence, the low energy effects of any such term are suppressed by powers of this scale and further characterized by an arbitrary dimensionless constant $c$, naively of order 1 . In the top-down approach one starts by assuming a specific theory of NP. If the generic mass $M$ of all new (beyond the SM) particles is larger than the electroweak scale, the effects of NP are described by an effective field theory (EFT). This corresponds to the enlarged Lagrangian of the bottom-up case, only now the coefficients of higher dimension operators are calculated in terms of underlying NP parameters. If, for example, the NP particles only enter at long distances through loops, then $\Lambda_{\mathrm{NP}} \sim 4 \pi M$.

The bottom-up approach is fairly general, encompassing many realistic extensions of the SM, that is, it is a model independent approach and has just the right number of necessary parameters. By contrast, the top-down approach is case specific and often has many more parameters than needed to describe the low energy physics (for example, the MSSM has over 100 parameters). On the other hand, the top-down approach gives correlations between short and long distance physics that, by design, are not available in bottom-up. Moreover, while both approaches give correlations among long distance processes, top-down often gives restrictions on the coefficients $c$ of higher dimension operators of the bottom-up approach that may result in additional low energy correlations. A reasonable way of organizing our thinking is this: do a bottom-up analysis first, to determine generic constraints on new physics. Then see if specific theories, or classes of them, satisfy these constraints and/or give additional correlations.

In this discussion we have kept $\Lambda_{\mathrm{NP}}$ generic, but if the NP has anything to do with the solution to the fine tuning problem, expect $\Lambda_{\mathrm{NP}} \sim 1 \mathrm{TeV}$. The flavor problem, arguably the reason for this annual meeting, can be seen from the following bottom-up argument. Consider the following $\Delta F=2$ FCNC operators (terms in the Lagrangian of the bottom-up approach)

$$
\frac{1}{\Lambda_{\mathrm{NP}}^{2}}\left[c_{1}\left(\bar{d}_{L} \gamma^{\mu} s_{L}\right)\left(\bar{d}_{L} \gamma_{\mu} s_{L}\right)+c_{2}\left(\bar{u}_{L} \gamma^{\mu} c_{L}\right)\left(\bar{u}_{L} \gamma_{\mu} c_{L}\right)+\text { h.c. }\right]
$$

\footnotetext{
${ }^{1}$ For that, see talk by M. Neubert in this volume
} 

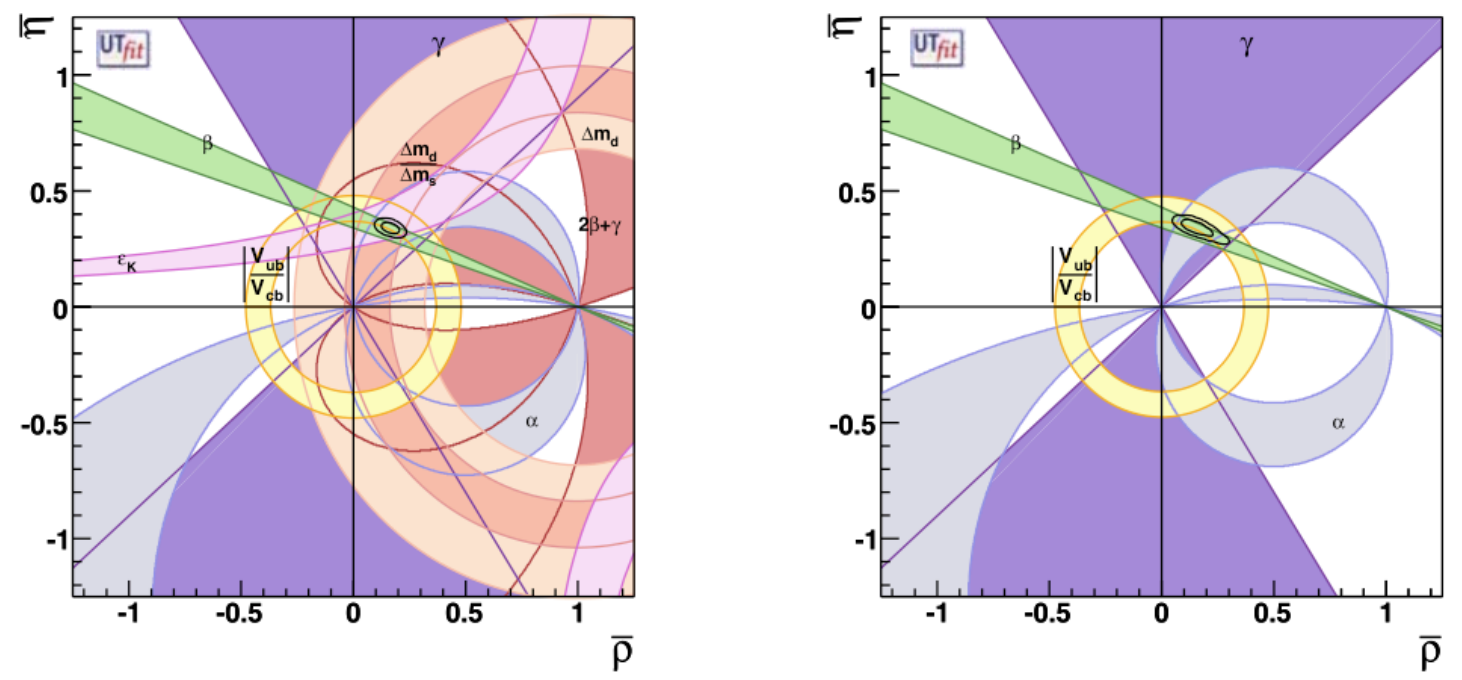

Figure 1: Comparison of the UTFit[11] determination of the apex of the unitarity triangle (UT) using (i) left panel: the measurements of $\left|V_{u b}\right| /\left|V_{c b}\right|, \varepsilon_{K}, \Delta m_{d}, \Delta m_{d} / \Delta m_{s}, \alpha, \beta, \gamma, \Delta \Gamma_{d} / \Gamma_{d}, \Delta \Gamma_{s} / \Gamma_{s}, A_{S L}^{d}$, and the dimuon asymmetry from D0, and (ii) right panel: only $\left|V_{u b}\right| /\left|V_{c b}\right|, \alpha, \beta$ and $\gamma$ are used. Assuming MFV These are largely unaffected by NP.

Ignoring electroweak FCNCs, neutral meson mixing data gives[1]

$$
c_{1}^{(\mathrm{data})}=(8.8+0.033 i) \times 10^{-7}\left(\frac{\Lambda_{\mathrm{NP}}}{1 \mathrm{TeV}}\right)^{2}, \quad c_{2}^{(\mathrm{data})}=(5.9+1.0 i) \times 10^{-7}\left(\frac{\Lambda_{\mathrm{NP}}}{1 \mathrm{TeV}}\right)^{2} .
$$

There being no reason to expect a cancellation between the electroweak and NP contributions, the couplings $c_{i}$ should be smaller than $c_{i}^{(\mathrm{data})}$. The problem is that this requires extraordinarily small dimensionless couplings.

I will argue that

1. Combining the Minimal Flavor Violation hypothesis (MFV) with the bottom-up approach is an effective way of explaining naturally the small numbers of the flavor problem.

2. Top-down approaches that successfully address the flavor problem give, with a few notable exceptions, a low energy EFT that satisfies the MFV hypothesis.

\section{Bottom-Up and Minimal Flavor Violation}

In the bottom-up approach we could just declare the $c_{i}$ 's to be as small as necessary. But if we have an organizing principle that explains the smallness of the FCNC couplings we may both find new correlations that test this principle and have a guide to constructing new theories that pass this test. It was realized in the early days of the MSSM that both real and imaginary parts of the $\Delta S=2$ FCNCs are naturally small if the only source of flavor change is the quark Yukawa couplings[2]. The idea was extended to non-SUSY theories[3] and eventually stated in a purely model independent, bottom-up approach[4]. In the absence of the Yukawa couplings $Y_{U, D, E}$, the 
SM has a large flavor symmetry, $G_{f}=S U(3)^{5}$ (times $U(1)$ factors). The SM is invarinat under $G_{f}$ if $Y_{U, D, E}$ are taken as spurions that transform under $G_{f}$. Minimal Flavor Violation (MFV) is the statement that the only sources of breaking of $G_{f}$ are the Yukawas. For the bottom-up approach one is led to construct $G_{f}$ symmetric higher dimension operators that include $Y_{U, D, E}$ in such a way that invariance under $G_{f}$ is maintained. The basic building blocks are the bilinears $\bar{Q}_{L} Y_{U} Y_{U}^{\dagger} Q_{L}$, $\bar{D}_{R} Y_{D}^{\dagger} Y_{U} Y_{U}^{\dagger} Q_{L}$ and $\bar{D}_{R} Y_{D}^{\dagger} Y_{D} D_{R}$, with appropriate Dirac-gamma matrices understood. Bilinears with additional powers of Yukawas can be constructed but give effects suppressed by additional factors of either small masses or small mixing angles.

Take a quark basis with $Y_{D}=\lambda_{d}$ and $Y_{U}=V^{\dagger} \lambda_{u}$, with $\lambda_{d, u}$ diagonal and where $V$ is the CKM matrix. Some operators allowed by symmetry are suppressed by powers of small Yukawa couplings. For low energy flavor changing processes one may work to linear order in $\lambda_{d}$ and neglect all but the combination $\left(\lambda_{F C}\right)_{i j}=\left(Y_{U} Y_{U}^{\dagger}\right)_{i j} \approx \lambda_{t}^{2} V_{3 i}^{*} V_{3 j}$ (with $i \neq j$ ). Buras further defines[5] Constrained MFV (CMFV) by restricting the operators of the effective theory to be the same as those that appear in the low energy limit of the SM. However, many common extensions of the SM that satisfy the MFV hypothesis are not CMFV. For example, in two higgs doublet models (2HDMs) at large $\tan \beta$ the $b$-quark Yukawa coupling cannot be neglected and therefore significant effects from additional operators arise. Several popular specific models incorporate CMFV, among them the 2HDM, both of type I or II, at small $\tan \beta$, the MSSM with MVF (including gauge mediated SUSY breaking) at small $\tan \beta$, minimal universal extra dimensions[6] and the littlest Higgs[7] with T-parity[8] with degenerate mirror fermions.

In any extension of the SM the determination of CKM matrix entries must be done anew since measurements may now depend on additional model parameters. $\left|V_{u s}\right|,\left|V_{c b}\right|,\left|V_{u b}\right|$ and the unitarity triangle angle $\gamma$ are normally unaffected since they are determined from tree level graphs. MFV has 11 parameters: 4 from the CKM plus the coefficients $c_{i} / \Lambda_{\mathrm{NP}}^{2}$ of 7 operators of dimension 6 . In MFV the unitarity triangle angles $\beta$ and $\alpha$ are also unaffected and therefore the apex of the unitarity triangle is essentially unchanged (see Fig. 1). For the new parameters one finds $\Lambda_{\mathrm{NP}} / \sqrt{\left|c_{i}\right|}>$ several TeV, the bound varying slightly according to which operator and the sign of $c_{i}$ (e.g., Fig. 2).

\subsection{Unconstrained (large $\tan \beta$ ) MFV}

In multi-higgs models it is not necessary that $\lambda_{b} \ll \lambda_{t}$. For example, $\lambda_{b} \geq \lambda_{t}$ in $2 \mathrm{HDM}$ or MSSM with $\tan \beta \geq m_{t} / m_{b}$. B physics already places significant bounds on the 2HDM parameter space (see Fig. 3). For unconstrained MFV (UMFV) the overall normalization of the Yukawa couplings is free and therefore we include some operators that are neglected in MFV. As a result the substantial correlation obtained in MFV between $K$ and $B$ amplitudes is weakened. Also, $\lambda_{b} \gtrsim 1$ affects helicity suppressed observables in $B$ physics. In particular $B \rightarrow \ell^{+} \ell^{-}$can be tremendously enhanced.

Whatever the UV completion of UMFV may be, it must contain at least two different EW order parameters, decoupling the source of mass of $U$-type quarks from that of $D$-type quarks. It is therefore standard to take the field content of the 2HDM for UMFV. The PQ symmetry (with, say, $H_{U}$ charged, and $H_{D}$ neutral) must be explicitly broken, else an unacceptably light axion will result. The breaking of PQ symmetry involves a new small parameter which we can treat as a spurion (just like the Yukawas, that break $G_{f}$ ). The most dangerous operators are in fact renormalizable Yukawa 

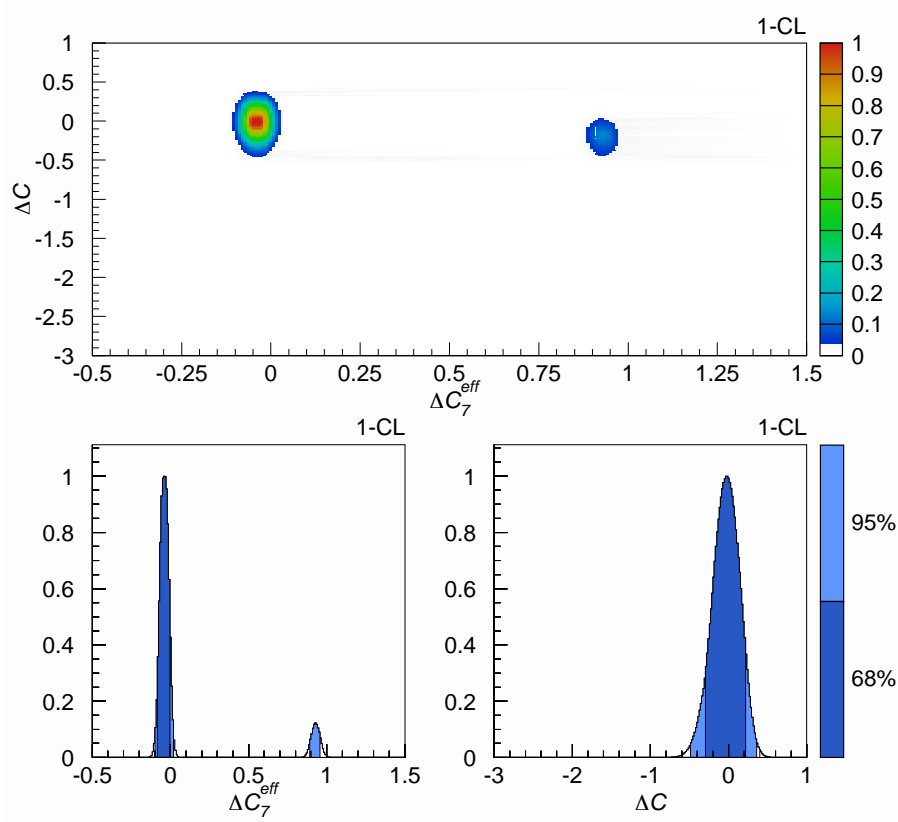

Figure 2: Sample state of the art constraints of MFV parameters, from Ref. [12]. $\Delta C_{7}^{\text {eff }}$ is related to the coefficient of the $b \rightarrow s$ transition magnetic operator, while $\Delta C$ is associated with operators like $\bar{q}_{L} Y_{U} Y_{U}^{\dagger} \gamma^{\mu} q_{L} H^{*} D_{\mu} H$ which can modify the $Z$ coupling to quarks. It is seen that the joint determination nearly excludes the reflection point at $\Delta C_{7}^{\text {eff }} \approx-2 C_{7}^{\text {eff,SM }}$.

couplings to the "wrong" higgs doublet, e.g.,

$$
\left(H_{U}^{*} i \tau^{2} \bar{Q}_{L}\right)\left(\varepsilon_{0}+\varepsilon_{1} Y_{U} Y_{U}^{\dagger}+\cdots\right) Y_{D} D_{R}+\text { h.c. }
$$

One finds, for example, that[4] for $\left|\varepsilon_{1}\right|=\varepsilon_{0} \gtrsim 0.2 / \tan \beta$ and $M_{H} \lesssim 10 / \tan \beta \mathrm{GeV}$ the rate for $B \rightarrow \ell^{+} \ell^{-}$is enhanced relative the SM by two orders of magnitude. The effects on $B \rightarrow \ell v$, $B \rightarrow X_{s} \gamma$ and $\Delta m_{B_{s}}$ are less pronounced, at the $10 \%-50 \%$ level. While other physics may give an enhancement in the helicity suppressed rate for $B \rightarrow \ell^{+} \ell^{-}$, strong evidence for UMFV would follow from enhancements in both $B_{s}$ and $B_{d}$ decays if, in addition, they satisfy $\Gamma\left(B_{s} \rightarrow \ell^{+} \ell^{-}\right) / \Gamma\left(B_{d} \rightarrow\right.$ $\left.\ell^{+} \ell^{-}\right)=\left|V_{t s} / V_{t d}\right|^{2}$. Note that the interactions in Eq. (2.1) are not mandated by symmetry. For example, one could break PQ symmetry by introducing an electroweak triplet or singlet with hypercharge +1 . The PQ symmetry breaking spurion would carry two units of PQ charge and the dangerous couplings in Eq. (2.1) would be avoided.

\subsection{NMFV and MFV-GUTs}

Several extensions of the SM get around the flavor problem without strictly adhering to the MFV hypothesis. Since we have no experimental proof of MFV we should keep an open mind and search for other organizing principles in a bottom-up approach. In next to MFV (NMFV) the MFV hypothesis is slightly relaxed by allowing arbitrary breaking of the flavor symmetry in operators that involve the third generation.[9] This works because the most stringent bounds on the scale of 


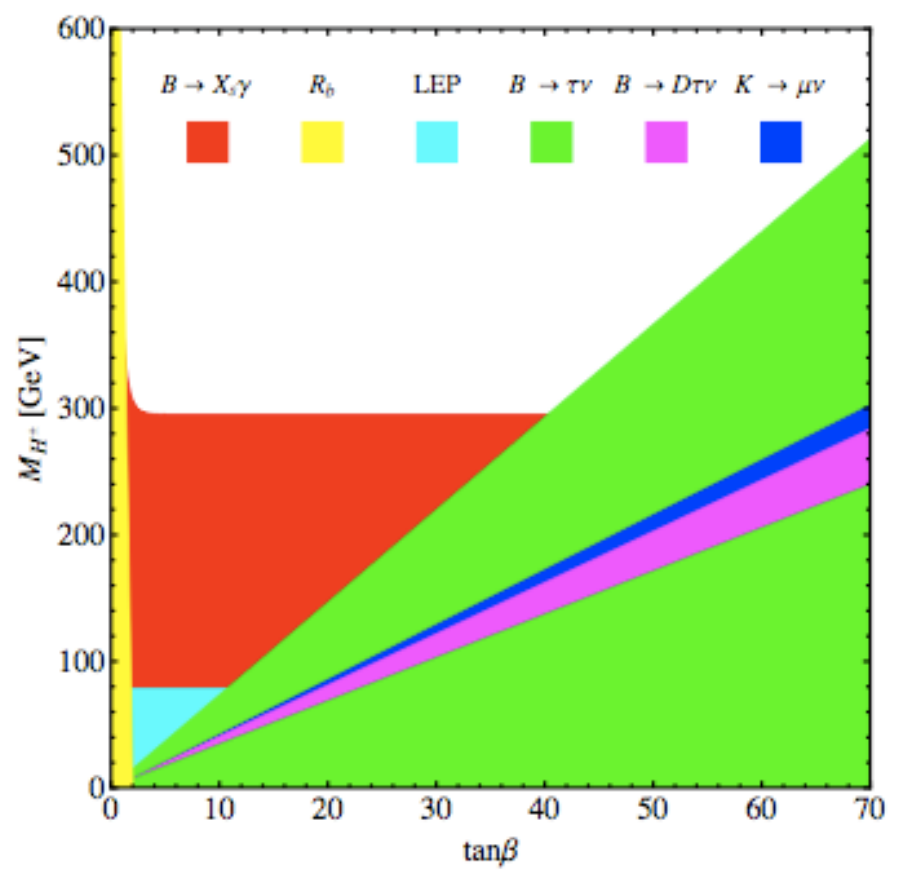

Figure 3: Excluded parameter space in the 2HDM. Except for a tiny sliver from LEP experiments, the exclusion is from (low energy) flavor physics[19]. Radiative $B$ decays plays a prominent role.

NP come from processes that involve the first two generations, as in the discussion of (1.1). The construction can be formalized in terms of symmetries: the NP respects a $S U(2)^{5}$ subgroup of $G_{f}$. Therefore the EFT can be again constructed using a spurion technique (for this and specific bounds see Ref. [10]).

MFV does not give enough CP violation for baryogenesis. However, the lepton sector counterpart, MLFV [13], easily accommodates the conditions for leptogenesis [14]. MLFV has no significant effect on B decays. However, the best justification we have for MFV for leptons is that the source of flavor breaking is common for quarks and leptons, originating in a GUT [15]. This can lead to additional correlations that may include some really novel phenomena, e.g., $\mu \rightarrow e \gamma$ and other lepton flavor changing processes.

Digression: Relations vs. Correlations We have been speaking throughout of "correlations" that arise from restricting the coefficients of the effective theory in the bottom-up approach. We also talk of correlations in the context of top-down, both among log distance effects and between long and short-distances. I do not know who started this usage, or when or in what context. The fact is that quantum field theory (QFT) produces "relations" between observables. Parameters of a QFT are not directly observable, they are crutches to produce relations between observables. These relations are fixed, that is, they are not statistical in nature. However, consider a model, like the MSSM, with many more parameters than the SM. Most of these parameters affect only marginally low energy observables, and we can think of them as slightly modifying the SM relations. A genuinely interesting and valid question is, in scanning over reasonable values of these parameters 
how large are the modifications to the SM relations? The answer to this question can be though of as giving a correlation, rather than a relation, between observables.

\section{Top-down and SUSY}

The flavor problem imposes severe constraints on models of NP that address the fine tuning problem. For this reason, many models are built to naturally exhibit MFV. To this extent, and given that we have already studied MFV in a model independent way, the questions are (i) how naturally is MFV incorporated in any particular model, and (ii) are there any additional restrictions/correlations from any particular model or class of models.

Not all NP models incorporate the MFV hypothesis. For some, like the general MSSM, with or without $\mathrm{R}$ parity, flavor is plainly a disaster, requiring arbitrary fine tuning of many parameters. Others, like the Lee-Wick extension of the SM, happily avoid dangerous FCNC without relying on the MFV hypothesis while gauge-mediated SUSY was invented to incorporate MFV automatically.

There are many SUSY based models, the MSSM, minimal SUGRA, gauge mediated SUSY, etc. In order to navigate this zoo, let us begin by specifying what is meant by the MSSM. Define the Minimal Supersymmetric Standard Model (MSSM) to have the field content and interactions of the supersymmetrized 2HDM, plus most general soft SUSY breaking terms. That is, the MSSM is SUSY Yang-Mills with the SM gauge group and with matter fields:

$\begin{array}{rlllllll}\text { spin-1/2: } & Q_{L} & U_{R} & D_{R} & L_{L} & E_{R} & \widetilde{H}_{U} & \widetilde{H}_{R} \\ \text { spin-0 : } & \widetilde{Q}_{L} & \widetilde{U}_{R} & \widetilde{D}_{R} & \widetilde{L}_{L} & \widetilde{E}_{R} & H_{U} & H_{R}\end{array}$

Fields adorned by a tilde are spin $\pm 1 / 2$ partners of the fields of the 2 HDM. There are in addition "gauginos," spin-1/2 partners of the gauge bosons. In addition to the interactions dictated by gaugeand super- symmetries, we have:

- Supersymmetric marginal interactions (with dimensionless couplings). For example, the Yukawa coupling $Y_{U} H_{U} \bar{Q}_{L} U_{R}$ is related to other Yukawas, like $Y_{U} \widetilde{U}_{R} \bar{Q}_{L} \widetilde{H}_{U}$, and to scalar quartics like $Y_{U}^{\dagger} Y_{U} \widetilde{U}_{R}^{\dagger} \widetilde{U}_{R} \widetilde{Q}_{L}^{\dagger} \widetilde{Q}_{L}$.

- One unique supersymmetric mass “ $\mu$-term:” $\mu^{2}\left(H_{U}^{\dagger} H_{U}+H_{D}^{\dagger} H_{D}\right)+\mu\left(\overline{\widetilde{H}}_{U} \widetilde{H}_{D}+\overline{\widetilde{H}}_{D} \widetilde{H}_{U}\right)$.

- Arbitrary (non-supersymmetric) relevant operators, or "soft-SUSY-breaking" interactions. These include scalar cubics that mimic the Yukawas, as in $A_{U} H_{U} \widetilde{Q}_{L}^{\dagger} \widetilde{U}_{R}$ and also scalar masses, as in $\tilde{m}_{Q_{L}}^{2} \widetilde{Q}_{L}^{\dagger} \widetilde{Q}_{L}$, and gaugino masses.

The Minimal in MSSM refers to the particle content. The class of interactions is motivated by $N=1$ supergravity mediated SUSY breaking with radiative EW breaking.

The MSSM is a flavor disaster. Neither squark mass matrices $\tilde{m}_{Q_{L}}^{2}, \tilde{m}_{D_{R}}^{2}, \tilde{m}_{D_{R}}^{2}$ nor cubics $A$ are aligned a priori with the quark mass matrices from the Yukawas $Y_{U}$ and $Y_{D}$, resulting in huge FCNCs. To be quantitative we introduce a measure for this, the fractional deviation from the unit matrix: $\delta=\Delta m^{2} / \bar{m}^{2}$. There are many entries to consider, by flavor, $U$ vs. $D$ and $L$ vs. $R$. For example, assuming common squark and gluino masses of $350 \mathrm{GeV},\left|\left(\delta_{12}^{d}\right)_{L R}\right|<5 \times 10^{-4}$, $\left|\left(\delta_{13}^{d}\right)_{L R}\right|<1 \times 10^{-2}$ and $\left|\left(\delta_{23}^{d}\right)_{L R}\right|<5 \times 10^{-3}$ (taken from [16], which also has additional detail). 
A semi-bottom-up approach to address this problem is to incorporate the MFV hypothesis into the MSSM. The supersymmetric couplings already satisfy this (they are either the flavor blind gauge couplings and $\mu$-term, or are written in terms of $Y_{U}$ and $Y_{D}$ ). Then MFV dictates

$$
\begin{aligned}
\tilde{m}_{Q_{L}}^{2} & =\tilde{m}_{0}^{2}\left(a_{1}+b_{1} Y_{U} Y_{U}^{\dagger}+b_{1}^{\prime} Y_{D} Y_{D}^{\dagger}+\cdots\right) \\
\tilde{m}_{U_{R}}^{2} & =\tilde{m}_{0}^{2}\left(a_{2}+b_{2} Y_{U} Y_{U}^{\dagger}+\cdots\right) \\
\tilde{m}_{D_{R}}^{2} & =\tilde{m}_{0}^{2}\left(a_{3}+b_{3} Y_{D} Y_{D}^{\dagger}+\cdots\right) \\
A_{U} & =A \tilde{m}_{0}\left(a_{4} Y_{U}+b_{4} Y_{D} Y_{D}^{\dagger} Y_{U}+\cdots\right) \\
A_{D} & =A \tilde{m}_{0}\left(a_{5} Y_{D}+b_{5} Y_{U} Y_{U}^{\dagger} Y_{D}+\cdots\right)
\end{aligned}
$$

Here $\tilde{m}_{0}$ is a mass parameter, and $a_{i}, b_{i}$ are dimensionless numbers which we assume are order 1 . Since we are interested in low energy phenomena (my assigned title says something about $B$ decays), we can now integrate out all of the SUSY partners ${ }^{2}$ to obtain an EFT for the 2HDM with MFV. At low to moderate $\tan \beta$ this is just the CMFV, else we have MFV at large $\tan \beta$. In either case the scale of new physics is $\Lambda \simeq 4 \pi \tilde{m}_{0}$ (there is a $4 \pi$ because the effects of SUSY particles come from diagrams of at least one loop). Given the bounds from MFV, SUSY-MFV is not experimentally excluded provided $\tilde{m}_{0} \gtrsim($ few $\mathrm{TeV}) / 4 \pi$.

Even though the MSSM is disastrous, it is a good place to start the SUSY discussion if nothing else because it gives us a template. It is general enough that the observable sectors of most SUSY models are special cases of the MSSM. Keep in mind, however, that there are models with more stuff, not in the MSSM.

First there are additional supersymmetric marginal interactions that were not included because they violate either lepton number or baryon number, or both. We can look past this SUSY embarrassment by artificially imposing a global discrete " $R$-parity" symmetry under which all tilde-fields and gauginos are odd. There is really interesting flavor physics associated with these $R$-parity breaking interactions. For example, the proton can be stabilized if terms of the form $U_{R} D_{R} D_{R}$ are excluded by imposing $B$-parity. Then lepton number violating superpotential terms $\sim Q_{L} L_{L} D_{L}^{c}$ can produce significant flavor changing effects[17]. These lead to loss of correlations among FCNC processes, and affect the extraction of CKM elements. Some effects arise in unexpected places, e.g., in the evolution of a shockwave both before and after rebound in a supernova[18].

Then there are models of interest for which there are additional fields that couple to the observable sector. For example, additional neutral fields are often added in addressing the $\mu$-problem (why is $\mu$ of the order of $\tilde{m}_{0} \sim$ EW-scale?). In many cases these additional fields are flavor blind and their quantum numbers preclude direct coupling to quarks and leptons, so there are minimal modification to flavor physics. In some cases the additional physics is intrinsically tied to flavor physics, as is the case of Littlest-Higgs assisted SUSY models. There is a bewildering array of possibilities.

So let us concentrate on the MSSM. We have shown how the MFV assumption cures the MSSM's flavor troubles. Generally this leaves us, at low energies, with an EFT that incorporates MFV. Interestingly, one can relax the artificially imposed $R$-parity symmetry if one is willing to apply the MFV hypothesis to renormalizable couplings. Baryon and lepton numbers are violated but at levels consistent with present bounds[20].

\footnotetext{
${ }^{2}$ Except, perhaps, the LSP and maybe the NLSP
} 
That's as much as we can do with a bottom-up approach. Let's turn to top-down. All models begin by assuming one has the field content of the MSSM (extended perhaps for grand-unification) and interactions that respect supersymmetry. One then has to figure out how to break SUSY. In all cases one introduces additional fields (a new "SUSY-breaking" or "hidden" sector) that force the vacuum (or long-lived metastable state) to be non-SUSY invariant. This breaking needs be communicated to the MSSM fields (the "observable sector"). The minimal choice is by (super)gravitational interactions: we know they exist and couple universally, that is, to both observable and hidden sectors. Among the alternatives, that introduce additional fields to communicate the breaking of SUSY, Gauge Mediation is the most popular.

Supergravity mediated SUSY-breaking gives effectively (once the hidden sector is integrated out) the MSSM. Since gravitational interactions break all global symmetries, it gives in fact the MSSM with all of its flavor issues and without $R$-parity. I am not sure why there is still interest in this theory. Perhaps it is because the mechanism[22] of electroweak symmetry breaking is really beautiful: the higgs's matrix of squared-masses develops a negative eigenvalue as it is RGE evolved from the Planck scale down to the EW scale. Or perhaps it is the belief that the problems may be solved by new undisclosed Plank scale physics. Certainly to proceed we must make further assumptions that will seem unnatural in the absence of such new very UV physics.

The simplest is the MFV hypothesis. This results in the MSSM with MFV (and approximate $R$ parity). There are two other very popular approaches. The constrained MSSM (CMSSM) assumes universality of the soft SUSY breaking parameters and GUT relations among them. The soft SUSYbreaking parameters are now given as in Eqs. (3.1) but are computable. There are only 4 new paramters (beyond the SM's): $\tilde{m}_{0}$ and $A$ in Eqs. (3.1), the gaugino mass scale $\tilde{m}_{1 / 2}$ and the $\mu$ parameter (of the SUSY $\mu$-term). The small number of free parameters makes this a very (most?) popular version of the MSSM among phenomenologists. If we give up grandunification relations but insist on soft SUSY-breaking terms universality we obtain the next-to-CMSSM (NC-MSSM). It still has a restricted form of Eqs. (3.1) but does have more free parameters than the CMSSM. All these approaches give versions of MFV at low energies, with varying numbers of additional shortlong and long-long distance correlations. The reason the CMSSM and the NC-MSSM are popular is the tractability afforded by a limited number of underlying parameters. Since there is nothing fundamental about them I believe practitioners would drop the restricted approaches without an afterthought if and when it becomes phenomenologically indicated.

One may try to derive MFV. For example, one may use gauged horizontal interactions to produce flavor and construct the model so that it effectively gives MFV. In Ref. [23] only an SU(2) horizontal symmetry is used giving, I believe, an example of NMFV.

While gauge-mediation[24], anomaly-mediation[25], etc, require that we postulate the existence of additional fields (beyond the MSSM and gravity), they are attractive because the solution to the MSSM flavor problem is natural. These theories almost always ${ }^{3}$ lead to an effective MSSM sector with MFV. In the absence of the hidden sector one simply has the supersymmetrized SM which in a trivial way satisfies the MFV hypothesis. In gauge mediation there is no direct coupling of the fields in the hidden sector that break SUSY to SM fields. There is a set of "mediators," fields that carry SM gauge quantum numbers and couple to SUSY breaking fields of the hidden sector.

\footnotetext{
${ }^{3}$ But not always. MFV is not necessary, it depends on the nature of the mediators.
} 
Hence SUSY breaking is communicated through flavor blind gauge interactions. Flavor-dangerous non-renormalizable interactions coupling the two sectors directly are suppressed by powers of a very large scale. ${ }^{4}$

We are driven to conclude that but for a few exceptions the low energy description of SUSY is that of MFV.

\section{References}

[1] These numbers are taken from the talks by Nir, Perez and Weiler and Plank09, Padova, May 2009

[2] R. Barbieri and R. Gatto, Phys. Lett. B 110, 211 (1982). J. R. Ellis and D. V. Nanopoulos, Phys. Lett. B 110, 44 (1982). M. Dugan, B. Grinstein and L. J. Hall, Nucl. Phys. B 255, 413 (1985).

[3] R. S. Chivukula and H. Georgi, Phys. Lett. B 188, 99 (1987).

[4] G. D’Ambrosio, G. F. Giudice, G. Isidori and A. Strumia, Nucl. Phys. B 645, 155 (2002) A. J. Buras, P. Gambino, M. Gorbahn, S. Jager and L. Silvestrini, Phys. Lett. B 500, 161 (2001)

[5] A. J. Buras, Acta Phys. Polon. B 34, 5615 (2003)

[6] T. Appelquist, H. C. Cheng and B. A. Dobrescu, Phys. Rev. D 64 (2001) 035002

[7] N. Arkani-Hamed, A. G. Cohen, E. Katz and A. E. Nelson, JHEP 0207, 034 (2002)

[8] H. C. Cheng and I. Low, JHEP 0408, 061 (2004) H. C. Cheng and I. Low, JHEP 0309, 051 (2003)

[9] K. Agashe et al, "Next to minimal flavor violation," arXiv:hep-ph/0509117.

[10] T. Feldmann and T. Mannel, JHEP 0702, 067 (2007)

[11] M. Bona et al. (UTfit Collaboration), Phys. Rev. Lett. 97, 151803 (2006); see also http://www.utfit.org

[12] U. Haisch and A. Weiler, Phys. Rev. D 76, 074027 (2007)

[13] V. Cirigliano, B. Grinstein, G. Isidori and M. B. Wise, Nucl. Phys. B 728, 121 (2005) V. Cirigliano and B. Grinstein, Nucl. Phys. B 752, 18 (2006)

[14] V. Cirigliano, G. Isidori and V. Porretti, Nucl. Phys. B 763, 228 (2007)

[15] B. Grinstein, V. Cirigliano, G. Isidori and M. B. Wise, Nucl. Phys. B 763, 35 (2007)

[16] M. Artuso et al., Eur. Phys. J. C 57, 309 (2008)

[17] V. D. Barger, G. F. Giudice and T. Han, Phys. Rev. D 40, 2987 (1989).

[18] P. S. Amanik, G. M. Fuller and B. Grinstein, Astropart. Phys. 24, 160 (2005)

[19] The graphic is taken from the talk by Mikihiko Nakao, Rencontres de Moriond EW 2009.

[20] E. Nikolidakis and C. Smith, Phys. Rev. D 77, 015021 (2008)

[21] K. A. Intriligator, N. Seiberg and D. Shih, JHEP 0604, 021 (2006)

[22] L. Alvarez-Gaume, M. Claudson and M. B. Wise, Nucl. Phys. B 207, 96 (1982). L. Alvarez-Gaume, J. Polchinski and M. B. Wise, Nucl. Phys. B 221, 495 (1983).

[23] M. Dine, R. G. Leigh and A. Kagan, Phys. Rev. D 48, 4269 (1993)

[24] M. Dine and A. E. Nelson, Phys. Rev. D 48, 1277 (1993)

[25] L. Randall and R. Sundrum, Nucl. Phys. B 557, 79 (1999)

\footnotetext{
${ }^{4}$ This is true also of the supergravity models, but there the suppression is ineffective because the scale at which SUSY breaks is necessarily higher.
} 